\title{
Large-cell neuroendocrine carcinoma of the lung: A clinicopathologic study of eighteen cases and the efficacy of adjuvant treatment with octreotide
}

\author{
Pier Luigi Filosso, $\mathrm{MD}^{\mathrm{a}}$ \\ Enrico Ruffini, $\mathrm{MD}^{\mathrm{a}}$ \\ Alberto Oliaro, MD \\ Ottavio Rena, MD ${ }^{\mathrm{b}}$ \\ Caterina Casadio, $\mathrm{MD}^{\mathrm{b}}$ \\ Maurizio Mancuso, $\mathrm{MD}^{\mathrm{a}}$ \\ Davide Turello, MD \\ Riccardo Carlo Cristofori, MD \\ Giuliano Maggi, MD
}

From the Department of Thoracic Surgery, San Giovanni Battista Hospital, University of Torino, Torino, Italy, ${ }^{\mathrm{a}}$ and Service of Thoracic Surgery, Amedeo Avogadro University, Novara, Italy. ${ }^{\text {b }}$

Received for publication Jan 14, 2004; revisions received April 25, 2004; accepted for publication May 10, 2004

Address for reprints: Pier Luigi Filosso, MD, University of Torino Italy, San Giovanni Battista Hospital, Department of Thoracic Surgery, Via Genova, 310126 Torino, Italy (Email: pierluigifilosso@tiscalinet.it).

J Thorac Cardiovasc Surg 2005;129:819-24 $0022-5223 / \$ 30.00$

Copyright () 2005 by The American Association for Thoracic Surgery

doi:10.1016/j.jtcvs.2004.05.023
Objective: This study was undertaken to evaluate the efficacy of a new adjuvant protocol with octreotide, alone or in combination with radiotherapy, in radically resected large cell neuroendocrine carcinomas of the lung.

Methods: Between 1990 and 2001, a total of 18 consecutive patients affected by large cell neuroendocrine carcinomas of the lung were operated on. Lobectomy and systemic lymphadenectomy were performed in all cases. Postoperative radiotherapy was performed when stage was higher than Ib. Ten patients with positive results of preoperative indium In-111 pentetreotide scintigraphy received octreotide after the operation.

Results: Nine patients (50\%) had local recurrences or distant metastases (mean recurrence time 14 months); palliative chemotherapy was given, but all patients died. In 10 cases $(55.5 \%)$ octreotide alone or in combination with radiotherapy was administered as adjuvant treatment; 9 of these patients (90\%) are alive and free of disease $(P=.0007)$, and the other had liver and brain metastases 21 months after surgery.

Conclusions: Our preliminary results seem to demonstrate the efficacy of octreotide as adjuvant therapy in large cell neuroendocrine carcinomas of the lung when results of preoperative indium In-111 pentetreotide scintigraphy were positive. Further study are required to assess the utility of octreotide in patients with negative results of indium In-111 pentetreotide scintigraphy.

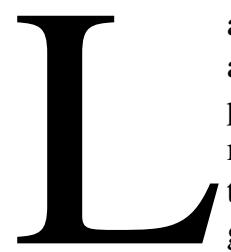

arge-cell neuroendocrine carcinoma (LCNC) of the lung represents about $2 \%$ to $3 \%$ of lung malignancies and it is known for its poor prognosis. ${ }^{1,2}$ The recent Travis classification of neuroendocrine tumors of the lung ${ }^{3}$ recognizes four types of neuroendocrine tumors: typical and atypical carcinoid, respectively low- and intermediategrade tumors, and LCNC and small cell lung carcinoma, high-grade neuroendocrine malignancies. Histologic criteria ${ }^{3}$ to diagnose LCNC are as follows: (1) a tumor with neuroendocrine morphologic appearance at light microscopy (organelle nesting, palisading, rosettes, and trabeculae), (2) high mitotic rate ( $\geq 11$ events $/ \mathrm{mm}^{2}$ ), (3) the presence of necrosis (centrilobular or in form of large areas), (4) the presence of large cell size, low nuclear to cytoplasmic ratio, vesicular 
chromatin, and frequent prominent nuclei, and (5) positive immunohistochemical staining for one or more neuroendocrine markers (neuron-specific enolase [NSE], chromogranin A, synaptophysin).

LCNCs of the lung are characterized by early relapses even in case of radical surgical resection. At this time it is not clear how affected patients should be treated. Surgery is considered the primary therapeutic option, usually followed by radiotherapy in case of T3 or N1-2 disease. Chemotherapy is proposed as palliative treatment when relapses are diagnosed, but survival does not appear to be influenced by this treatment. Somatostatin inhibits the secretion of a broad range of hormones, including growth hormone, insulin, glucagon, and gastrin. Octreotide is an 8-amino acid longacting somatostatin synthetic analog characterized by a remarkable biologic stability, higher than that of somatostatin, and biologic activities that overlap those of the native hormone. Its antitumoral effects are obtained by the inhibitions of neoplastic cell growth and angiogenesis, which have been well demonstrated in vitro. Octreotide's effects are mediated by somatostatin receptor subtype (sst) 2 and 5 activation, which results in inhibition of adenyl cyclase, decreased conductance of voltage-sensitive calcium channels, activation of potassium channels, and stimulation of tyrosine phosphatase activity. ${ }^{4}$

Octreotide is effective in the management of acute manifestations of the carcinoid syndrome. Octreotide is routinely used in neuroendocrine tumors of the gut, whereas at present experience with lung neoplasms with neuroendocrine features remains limited. We report a clinicopathologic study of 18 consecutive cases of radically resected LCNC of the lung; in 10 of them octreotide was administered alone or in combination with radiotherapy as adjuvant treatment. The aim of this preliminary study was to evaluate the effectiveness of this new adjuvant protocol in reducing tumor recurrence, thus prolonging survival.

\section{Material and Methods}

Between 1990 and 2001, a total of 1580 patients underwent radical resection of bronchogenic carcinoma at our department. Of these, $18(1.2 \%)$ were operated on for LCNC. Surgical specimens were all retrospectively reviewed by our pathologists, and diagnosis of LCNC was achieved according to the 1999 criteria of Travis and colleagues. ${ }^{3}$ Immunohistochemical stains included the general neuroendocrine markers, with polyclonal antibodies to synaptophysin (DAKO A/S, Glostrup, Denmark), chromogranin A (Roche Diagnostics GmbH, Mannheim, Germany), and NSE (DAKO $\mathrm{A} / \mathrm{S}$ ). For each antibody the percentage of positive cells (0 for negative, $1+$ for $1 \%-25 \%, 2+$ for $26 \%-50 \%, 3+$ for $>50 \%$ ) and the intensity of staining ( 0 for negative, 1 for weak, 2 for moderate, and 3 for strong) were recorded. Immunohistologic neuroendocrine markers were considered positive if more than $10 \%$ of neoplastic cells reacted with an intensity of $2+$ or greater. Three other patients were excluded from this study because they were affected by combined tumor (LCNC and adenocarcinoma or squamous cell carcinoma).

There were 11 male (61\%) and 7 female patients; mean age was 63 years (range 48-71 years). Patients' clinical characteristics are shown in Tables 1 and 2. At the time of presentation, 4 patients $(22.2 \%)$ were free of symptoms, with the tumor incidentally discovered. In the other 14 cases, symptoms at presentation were as follows: chest pain $(n=5)$, hemoptysis $(n=5)$, dyspnea $(n=4)$, weigh loss $(n=3)$, and persistent cough $(n=7)$. Thoracic computed tomographic scan was performed in all cases; chest wall invasion was evident in 2 patients $(11.1 \%)$ and confirmed at intervention and mediastinal lymph node enlargement in 1 (5.5\%). Transthoracic fine-needle aspiration biopsy was performed in 6 cases $(33.3 \%)$. Diagnosis of non-small cell lung carcinoma (NSCLC) was achieved in 5 cases $(83.3 \%)$, the remaining biopsy yielded inconclusive results. Bronchoscopy was performed in all patients; the diagnosis of NSCLC was made in 5 (27.7\%), and the diagnosis of atypical bronchial carcinoid was made in $1(5.5 \%)$. Preoperative serum neoplastic markers (carcinoembryonic antigen, tissue polypeptide antigen, NSE, chromogranin A) were recorded when available. Because it was available in our hospital, indium In-111 pentetreotide scintigraphy (OctreoScan; Mallinckrodt Inc, Hazelwood, Mo) was routinely performed in the preoperative assessment and follow-up of all patients with suspected or proven neuroendocrine tumor of the chest. Ten patients $(55.5 \%)$ were submitted to OctreoScan because of high NSE and chromogranin A serum levels (just because of the high probability of tumors with neuroendocrine features) or preoperative diagnosis of neuroendocrine tumor. Preoperative OctreoScan showed pathologic uptake corresponding to the tumor only.

\section{Follow-up}

All patients were directly followed up at our institution with periodic outpatient clinics. Data regarding survival and presence, type, and site of recurrence were recorded. No patients were unavailable for follow-up.

\section{Statistical Analysis}

Survival analysis was performed with the Kaplan-Meier curve. Differences between survival curves were computed with the logrank test. Statistical analysis were performed with STATISTICA release 6 software (StatSoft Inc, Tulsa, Okla).

\section{Results}

All patients underwent radical tumor resection, and lobectomy and systemic lymphadenectomy were performed in all cases. In 2 cases en bloc chest wall resection was required. One patient underwent mediastinoscopy, with histologic diagnosis of lymph node metastasis of atypical carcinoid; this patient underwent a left upper lobectomy with a definitive histologic diagnosis of pT2 N2 LCNC.

We adopted the 1997 revised TNM system ${ }^{5}$ for classification of LCNC. Median survival was 42 months. The 3and 5-year survivals were $63 \%$ and $35 \%$, respectively (Figure 1).

Somatostatin receptors (sst2 and sst5) were detected by polymerase chain reaction technique in all patients with 
TABLE 1. Clinical characteristics and patient outcomes

\begin{tabular}{|c|c|c|c|c|c|c|c|c|c|}
\hline $\begin{array}{c}\text { Age } \\
\text { (y) }\end{array}$ & Sex & Intervention & TNM & Site of recurrence & $\begin{array}{l}\text { Recurrence time } \\
\text { (mo) }\end{array}$ & $\begin{array}{c}\text { Survival } \\
\text { (mo) }\end{array}$ & $\begin{array}{l}\text { Survival after } \\
\text { recurrence } \\
\text { (mo) }\end{array}$ & Octreotide & Outcome \\
\hline 63 & $\mathrm{M}$ & RUL & T2 No & Mediastinum, liver & 38 & 48 & 10 & & Dead \\
\hline 70 & $M$ & LLL & T2 No & Brain & 14 & 19 & 5 & & Dead \\
\hline 66 & $\mathrm{~F}$ & LUL & T2 N1 & None & & 50 & & Yes & Alive \\
\hline 52 & $M$ & $\mathrm{LLL}+$ ribs & T3 N1 & Liver & 19 & 24 & 5 & & Dead \\
\hline 48 & $M$ & RUL & T2 N1 & None & & 43 & & Yes & Alive \\
\hline 58 & $\mathrm{~F}$ & RUL + ribs & T3 NO & Chest wall & 27 & 38 & 11 & & Dead \\
\hline 66 & $\mathrm{~F}$ & LUL & T2 N1 & None & & 61 & & Yes & Alive \\
\hline 68 & $M$ & LLL & $\mathrm{T} 2 \mathrm{~N} 2$ & Adrenal & 9 & 11 & 2 & & Dead \\
\hline 71 & $M$ & RLL & T2 N1 & None & & 44 & & Yes & Alive \\
\hline 70 & $M$ & RLL & T2 N1 & None & & 50 & & Yes & Alive \\
\hline 65 & $M$ & LUL & T2 N2 & None & & 23 & & Yes & Alive \\
\hline 66 & $\mathrm{~F}$ & RLL & T2 N2 & Brain & 12 & 16 & 4 & & Dead \\
\hline 54 & $\mathrm{~F}$ & RUL & T2 No & brain & 31 & 42 & 11 & & Dead \\
\hline 58 & $\mathrm{M}$ & LUL & T2 N1 & Liver, adrenal & 10 & 18 & 8 & & Dead \\
\hline 56 & $\mathrm{~F}$ & LUL & T2 N2 & Liver, brain & 21 & 32 & 11 & Yes & Dead \\
\hline 67 & $M$ & RLL & T2 No & None & & 32 & & Yes & Alive \\
\hline 59 & $M$ & RUL & T2 N1 & None & & 41 & & Yes & Alive \\
\hline 62 & $\mathrm{M}$ & RLL & T2 No & None & & 13 & & Yes & Alive \\
\hline
\end{tabular}

All patients who are listed as dead died of their disease. $R U L$, Right upper lobectomy; $R L L$, right lower lobectomy; $L L L$, left lower lobectomy; $L U L$, left upper lobectomy.

positive results of preoperative OctreoScan, and results were positive in all cases. Adjuvant therapy (radiotherapy) was administered to all patients with a TNM pathologic stage higher than $\mathrm{Ib}$, according to our oncologists' adjuvant protocol. Postoperative radiotherapy (54 Gy in 2-Gy daily fractions) was delivered with a $10-\mathrm{MeV}$ linear accelerator on the mediastinum (in case of $\mathrm{N} 1$ or N2 disease), chest wall (in case of T3 tumors), or both. No adjuvant chemotherapy was administered. In 10 patients $(55.5 \%)$, those in whom preoperative OctreoScan results were positive, adjuvant medical therapy with long-acting octreotide formulation (Sandostatina LAR $20 \mathrm{mg}$; Novartis Pharma, Milan, Italy) at $20 \mathrm{mg}$ every 28 days was administered alone (in patients with stage $\mathrm{Ib}$ disease) or in combination with radiotherapy (in patients with stage higher than $\mathrm{Ib}$ ). Patients received octreotide 1 month after surgery, and they are still continuing this therapy. Nine of 10 patients $(90 \%)$ are alive and do not show tumor recurrence $(P=.0007$; Figure 2$)$; the other had tumor recurrences in the liver and in the brain 21 months after a left upper lobectomy for pT2 N2 LCNC, and she survived 32 months after surgery. Octreotide was well tolerated in all cases, with only pain at the site of injection, transient nausea, abdominal cramps, and sometimes diarrhea recorded. No patients stopped the therapy. Follow-up was planned with chest radiographs every 3 months and thoracic, upper abdomen, and brain computed tomographic scan every 6 months. In recent years we have routinely used
Table 2. Patient characteristics

\begin{tabular}{lrc}
\hline & No. & $\%$ \\
\hline Sex & & \\
$\quad$ Male & 11 & 61 \\
$\quad$ Female & 7 & 39 \\
Side of the tumor & & \\
$\quad$ Left & 8 & 44.5 \\
$\quad$ Right & 10 & 55.5 \\
Smoking history & & \\
$\quad$ Yes & 14 & 77.7 \\
No & 4 & 22.3 \\
Stage & & \\
Ib & 5 & 27.8 \\
IIb & 8 & 27.4 \\
IIla & 5 & \\
\hline
\end{tabular}

OctreoScan and neoplastic serum markers (NSE and chromogranin A) in all cases.

Tumor relapses occurred in 9 patients: 8 of these (88.8\%) had not received octreotide as adjuvant therapy. Mean recurrence time was 19 months after surgery (range 9-41 months). OctreoScan was effective in detecting the recurrence sites in all patients. Palliative chemotherapy was proposed for all patients with tumor recurrence; cisplatin (or carboplatin) was administered in association with etoposide 


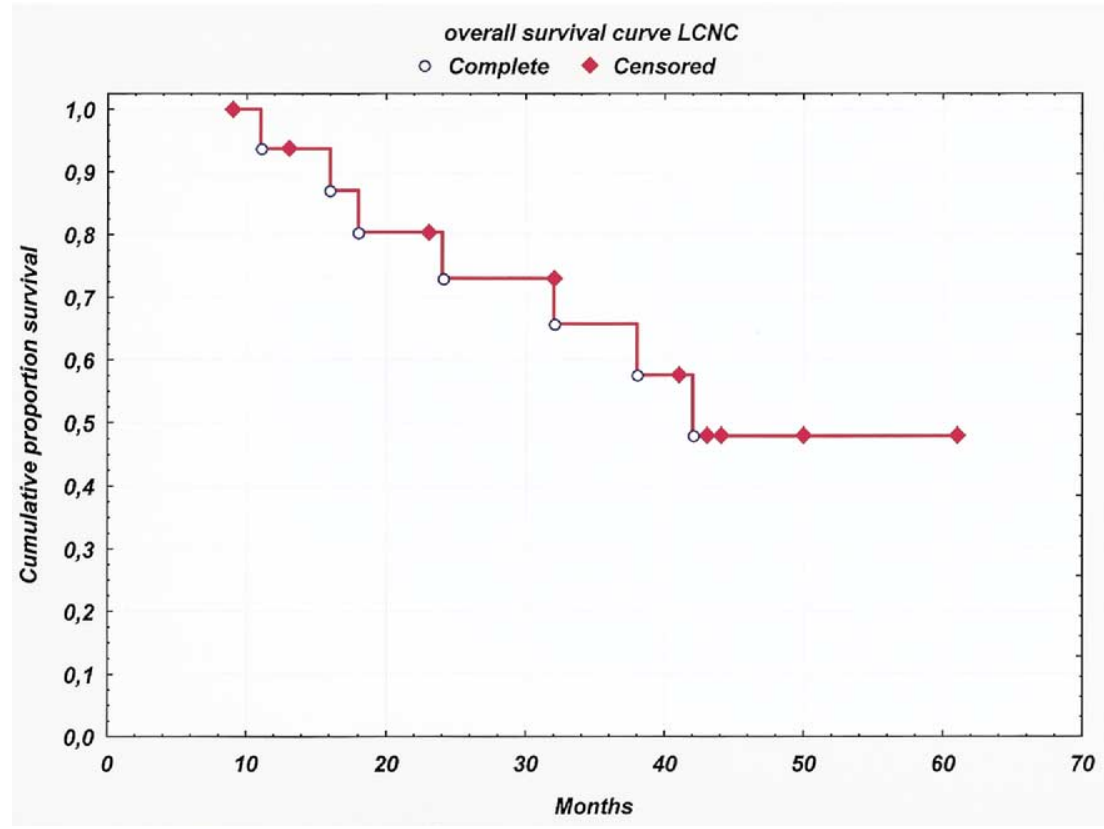

Figure 1. Overall survival curve.

Survival curve: octreotide vs, not octreotide - Complete + Censored

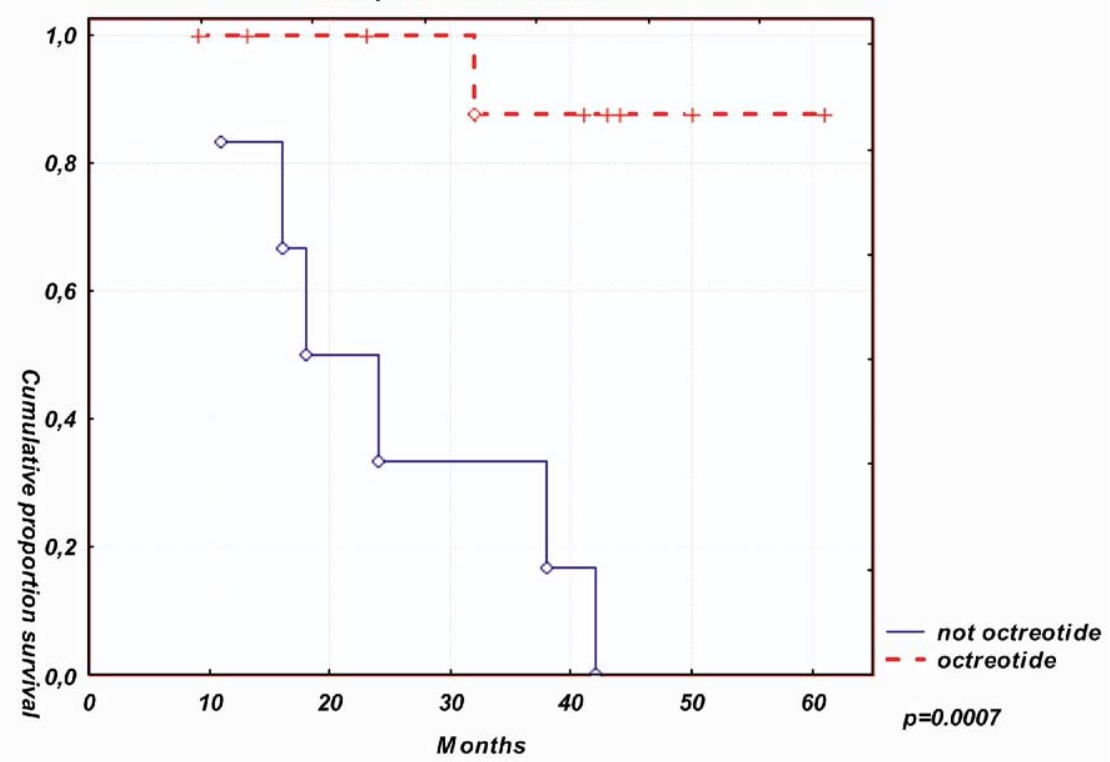

Figure 2. Comparison of survival curves with and without adjuvant medical treatment with octreotide $(P=.0007)$

or taxol. Mean survival after the metastatic spread was 8 months (range 2-11 months).

\section{Discussion}

In the 1999 World Health Organization classification of lung tumors, ${ }^{3}$ LCNC and small cell neuroendocrine carci- noma represent the high-grade entities among neuroendocrine neoplasms. The aggressive clinical behavior of LCNC is underlined by its poor prognosis and its high frequency of local or systemic recurrence after radical resection, even in early stages. ${ }^{2,6-9}$ Dresler and colleagues ${ }^{2}$ reported that stage I LCNC has an 18\% 5-year survival, and Jiang and associ- 
ates ${ }^{6}$ confirmed in their study the significantly poorer prognosis of LCNC relative to other NSCLCs. Our experience confirms these data; nevertheless octreotide, alone or in association with radiotherapy, was effective in prolonging survival and reducing the risk of tumor recurrence relative to radiotherapy alone.

The optimal therapeutic treatment for LCNC of the lung is still debated. Surgery remains the first therapeutic choice, but surgery alone is ineffective to prolong survival, and there is little consensus about the most effective adjuvant therapy. Postoperative chemotherapy (cisplatin-based for 4-6 courses) was firstly proposed by Dresler and colleagues, ${ }^{2}$ but even in stage I it did not improve survival. The insensitivity to chemotherapy of LCNC was suggested by Lai and associates in 1989. ${ }^{10}$ They examined multiple-drug resistance gene (MDR1) expression in a variety of lung cancers. This gene is frequently expressed in neoplasm that are chemoresistant or that have acquired chemoresistence. They observed that most MDRl gene expression was in the subgroup of lung cancers with neuroendocrine features. No significant data are available about the effectiveness of adjuvant chemotherapy in NSCLC, ${ }^{11}$ but Shepherd and coworkers $^{12}$ reported that adjuvant chemotherapy resulted in prolonged survival for many patients with stage I small cell lung carcinoma. Because of a part of the neuroendocrine spectrum of lung tumors, Iyoda and colleagues ${ }^{13}$ proposed adjuvant chemotherapy (cisplatin, carboplatin, or cyclophosphamide based) for large cell carcinomas with neuroendocrine features, and they concluded that this regimen might be effective in prolonging survival of patients with early stage large cell carcinoma with neuroendocrine features. However, adjuvant chemotherapy was not so effective in cases of advanced disease.

Postoperative radiotherapy is generally administered in advanced stages. We routinely administer adjuvant radiotherapy (54 Gy) for LCNC higher than stage I, according to our oncologists' protocol. Our preliminary study demonstrated the efficacy of this new adjuvant protocol including octreotide alone or in combination with radiotherapy: it significantly reduced the risk of tumor recurrence and prolonged survival in patients with radically resected LCNC. We used a long-acting octreotide formulation, which is well tolerated and easily administered. Patients receiving octreotide had positive results of preoperative OctreoScan; because OctreoScan reveals tumors with expression of sst2 and sst5, this information can be used to guide the clinician when choosing medical therapy with octreotide. The presence of these receptors (in vivo octreotide targets) was demonstrated by polymerase chain reaction technique in surgical specimens as well. Because of technical difficulties, it was not possible to demonstrate the presence of sst2 and sst5 somatostatin receptors in pathologic specimens from patients in whom preoperative OctreoScan was not performed.

Somatostatin analogs of the new generation (octreotide, lanreotide) are the first-line drugs to achieve a good clinical control of hormone-mediated symptoms of carcinoid tumors. The antigrowth effect of octreotide has been demonstrated in vitro, ${ }^{14,15}$ but its effects on human tumors remain controversial. Somatostatin biologic effects are mediated through five different receptor subtypes (sst1-sst5) that have been cloned and characterized to date. Octreotide, the first somatostatin analog introduced for clinical use, binds with high affinity to sst 2 and sst 5 receptors, binds with moderate affinity to sst3, and does not bind at all to sst 1 and sst $4 .{ }^{15}$ Among the five somatostatin receptors subtypes, sst1, sst2, and sst5 have all been implicated in the mediation of the antiproliferative effect of somatostatin in vitro. ${ }^{16}$ Recent observations have demonstrated that octreotide inhibits the growth and induces apoptosis in neuroblastoma cells that express high-affinity somatostatin receptors. ${ }^{17}$ The identification of high-affinity somatostatin receptors in peritumoral veins has been described previously. ${ }^{18}$ There is evidence that tumor angiogenesis, which originates from and depends on peritumoral host vessels, is influenced negatively by somatostatin and somatostatinlike factors. ${ }^{19,20}$ Thus the efficacy of somatostatin analogs in tumor growth control could be explained with this biologic mechanism, although further in vitro and in vivo studies are required to confirm this hypothesis.

In 2002 we described the long-term survivals of 7 patients with metastatic (liver) atypical bronchial carcinoids for which octreotide was the only medical therapy. ${ }^{21} \mathrm{Re}-$ duction of $60 \%$ in liver metastasis size was observed in 2 cases, complete resolution was observed in 1, and complete disease clinical control was observed in all. These results are similar to others reported in literature, ${ }^{22-25}$ but the latter are related only to metastatic gastrointestinal neuroendocrine tumors. No other large series have been reported regarding the efficacy of octreotide in neuroendocrine lung neoplasms.

Only two other systemic treatments (chemotherapy and interferon) have been demonstrated to have an antitumoral activity in neuroendocrine tumors, and both are less tolerated than octreotide because of their important adverse effects. Combination chemotherapy with 5-fluorouracil and streptozotocin gives a response rate ranging from $16 \%$ to $30 \%$, but this therapy presents important gastrointestinal and renal toxicity, with a poor tolerability for the patient. ${ }^{26,27}$ Interferon seems to have an impact on tumoral growth similar to that of somatostatin analogs, but it necessitates three subcutaneous injections weekly and entails many adverse effects. ${ }^{28}$

On the contrary, in our experience octreotide was really well tolerated, with few adverse effects: transient dose- 
dependent gastrointestinal symptoms (nausea, abdominal cramps and sometimes diarrhea), pain at the site of injection, and cholesterol gallstone ( $1 \%$ symptomatic) have been observed in our experience. All patients tolerated octreotide, and none of them stopped the treatment. We strongly believe in the effectiveness of this biologic drug, and to our knowledge this is the first report of successful combined adjuvant treatment in LCNC of the lung.

In conclusion, LCNC is a relatively uncommon highgrade tumor of the lung, characterized by poor prognosis and high rates of recurrences even in early stages. Surgery alone is ineffective in prolonging survival, and an optimal adjuvant therapy has still not been identified. This is the first description of the effectiveness of octreotide, alone or in combination with radiotherapy, as adjuvant treatment in radically resected LCNC of the lung. According to our preliminary results, and despite the limitations of this study (small number of cases and retrospective design), we propose the routine use of this protocol as postoperative treatment, even if surgery has been radical.

\section{References}

1. Travis WD, Rush W, Flieder DB, Falk R, Fleming MV, Gal AA, et al. Survival analysis of 200 pulmonary neuroendocrine tumors with clarification of criteria for atypical carcinoid and its separation from typical carcinoid. Am J Surg Pathol. 1998;22:934-44.

2. Dresler CM, Ritter JH, Patterson GA, Ross E, Bailey MS, Wick MR. Clinical-pathologic analysis of 40 patients with large cell neuroendocrine carcinoma of the lung. Ann Thorac Surg. 1997;63:180-5.

3. Travis WD, Corrin B, Shimosato Y, Brambilla E. The histological typing of lung and pleural tumors. 3rd ed. Berlin: Springer Verlag; 1999.

4. Reisine T, Bell G. Molecular biology of somatostatin receptors. Endocr Rev. 1995;16:427-42.

5. Mountain CF. Revision in the international system for staging lung cancer. Chest. 1997;111:1710.

6. Jiang SX, Kameya T, Shoji M, Dobashi Y, Shinada J, Yoshimura H. Large cell neuroendocrine carcinoma of the lung: a histologic and immunohistochemical study of 22 cases. Am J Surg Pathol. 1998;22: 526-38.

7. Takei H, Asamura H, Maeshima A, Suzuki K, Kondo H, Niki T, et al. Large cell neuroendocrine carcinoma of the lung: a clinicopathologic study of eighty-seven cases. J Thorac Cardiovasc Surg. 2002;124:285-92.

8. Hage R, Seldenrijk K, de Bruin P, van Swieten H, van den Bosch J. Pulmonary large cell neuroendocrine carcinoma (LCNEC). Eur J Cardiothorac Surg. 2003;23:457-60.

9. Zacharias J, Nicholson AG, Ladas GP, Goldstraw P. Large cell neuroendocrine carcinoma and large cell carcinomas with neuroendocrine morphology of the lung: prognosis after complete resection and systematic nodal dissection. Ann Thorac Surg. 2003;75:348-52.

10. Lai SL, Goldstein LJ, Gottesman MM, Pastan I, Tsai CM, Johnson BE, et al. MDRI gene expression in lung cancer. J Natl Cancer Inst. 1989;81:1144-50.
11. Non-Small Cell Lung Cancer Collaborative Group. Chemotherapy in non-small cell lung cancer: a meta-analysis using updated data on individual patients from 52 randomised clinical trials. BMJ. 1995;311: 889-90.

12. Shepherd FA, Evans WK, Feld R, Young V, Patterson GA, Ginsberg $\mathrm{R}$, et al. Adjuvant chemotherapy following surgical resection for small-cell carcinoma of the lung. J Clin Oncol. 1988;6:832-8.

13. Iyoda A, Hiroshima K, Toyozaki T, Haga Y, Baba M, Fujisawa T, et al. Adjuvant chemotherapy for large cell carcinoma with neuroendocrine features. Cancer. 2001;92:1108-12.

14. Taylor JE, Bogden AE, Moreau JP, Coy DH. In vitro and in vivo inhibition of human small cell lung carcinoma (NCI-H69) growth by a somatostatin analogue. Biochem Biophys Res Commun. 1998;153: $81-6$.

15. Weckbecker G, Liu R, Tolcsvai L, Bruns C. Antiproliferative effects of the somatostatin analogue octreotide (SMS 201-995) on ZR-75-1 human breast cancer cells in vivo and in vitro. Cancer Res. 1992;52: 4973-8.

16. Lamberts SW, van der Lely AJ, de Herder WW, Hofland LJ. Octreotide. $N$ Engl J Med. 1996;334:246-54.

17. Buscail L, Esteve JP, Saint-Laurent N, Bertrand V, Reisine T, O'Carroll AM, et al. Inhibition of cell proliferation by the somatostatin analogue RC-160 is mediated by somatostatin receptor subtypes SSTR2 and SSTR5 through different mechanisms. Proc Natl Acad Sci U S A. 1995;92:1580-4.

18. Borgstrom P, Hassan M, Wassberg E, Refai E, Jonsson C, Larsson SA, et al. The somatostatin analogue octreotide inhibits neuroblastoma growth in vivo. Pediatr Res. 1999;46:328-32.

19. Denzler B, Reubi JC. Expression of somatostatin receptors in peritumoral veins of human tumors. Cancer. 1999;85:188-98.

20. Woltering EA, Barrie R, O'Dorisio TM, Arce D, Ure T, Cramer A, et al. Somatostatin analogues inhibit angiogenesis in the chick chorioallantoic membrane. J Surg Res. 1991;50:245-51.

21. Filosso PL, Ruffini E, Oliaro A, Papalia E, Donati G, Rena O. Long-term survival of atypical bronchial carcinoids with liver metastases, treated with octreotide. Eur J Cardiothorac Surg. 2002;21: 913-7.

22. Leong WL, Pasieka JL. Regression of metastatic carcinoid tumors with octreotide therapy: two case report and a review of the literature. J Surg Oncol. 2002;79:180-7.

23. Ducreux M, Ruszniewski P, Chayvialle JA, Blumberg J, Cloarec D, Michel $\mathrm{H}$, et al. The antitumoral effect of the long-acting somatostatin analog lanreotide in neuroendocrine tumors. Am $J$ Gastroenterol. 2000;95:3276-81.

24. Aparicio T, Ducreux M, Baudin E, Sabourin JC, De Baere T, Mitry E, et al. Antitumour activity of somatostatin analogues in progressive metastatic neuroendocrine tumours. Eur J Cancer. 2001;37:1014-9.

25. Wiedenmann B, Rath U, Radsch R, Becker F, Kommerell B. Tumor regression of an ileal carcinoid under the treatment with the somatostatin analogue SMS 201-995. Klin Wochenschr. 1988;66:75-7.

26. Haller DG, Schutt A, Dayal Y, et al. Chemotherapy for metastatic tumors: an ECOG phase II-III trial. Proc Am Soc Clin Oncol. 1990; 9:102.

27. Moertel CG. Karnofsky memorial lecture. An odyssey in the land of small tumors. J Clin Oncol.1987;5:1502-22.

28. Oberg K, Funa K, Alm G. Effects of leukocyte interferon on clinical symptoms and hormone levels in patients with mid-gut carcinoid tumors and carcinoid syndrome. N Engl J Med. 1983;309:129-33. 